\title{
Combinatorial libraries on the Web
}

\author{
Richard Peters and Robert S. Sikorski
}

If you ever thought of looking for Web resources on combinatorial libraries, save yourself a lot of time and start here (see table below). We were asked by the editors to find useful information on the World Wide Web

Richard Peters and Robert Sikorski are Howard Hughes Medical Institute fellows at Harvard Medical School and the National Cancer Institute, respectively(rpeters@vp3.med.harvard.edu and rss@nchgr.nih.gov). concerning the topic of combinatorial libraries. As usual, we used our MIRIAD software to retrieve, filter, and review a total of 850 documents. To our surprise, almost $90 \%$ of all the documents we found dealt with the topic of combinatorial numbers in mathematics. While mathematicians may rejoice at the depth of information on this topic in cyberspace, for those more biotechnology oriented, winnowing out the $10 \%$ of these documents that relate to your field is an arduous task. To get you started, we have selected the documents that will give you a solid overview of what is out there. These documents should provide links that will take you to greater depth on individual topics. In addition, because phage display is often used in combinatorial schemes involving in vivo selection, we added two of our favorite sites dealing with this topic as well. For those wanting to save time on this topic and others, please review the archive of all WWW guide documents at our site: http://www.MedsiteNavigator.com.

\section{Combinatorial chemistry products and services sites}

\section{Page name}

Combinatorial chemistry:

A strategy for the future

Chemical \& engineering news

\section{URL}

http://www.awod.com/netsci//ssues/July95/feature2.html

http://pubs.acs.org/hotartcl/cenear/960212/spawns.html

Solid support combinatorial chemistry in lead discovery

A database system for combinatorial
synthesis experiments

Information management architecture for combinatoriai chemistry

http://www.awod.com/netsci/lssues/July95/feature3.html

http://www.awod.com/netsci/lssues/July95/feature7.html

http://www.awod.com/netsci/lssues/July95/feature8.html

Growing phage/phage preps

Vector $\mathrm{db}$
http://cajal.biology.utah.edu/JorgLabHomePage/

Protocols/NonJorgProtocols/phagepreps.html

\section{Comments}

A white paper on combinatorial chemistry

A special report from Chemical \& Engineering News on new software systems for combinatorial chemistry, published in February 1996

A review of the use of solid support in combinatorial chemistry

Description of a database system for combinatorial chemistry experiments

Article describing the use of Chem-X software with a relational database for the management of information in combinatorial chemistry

Phage preparation protocols

An extensive database of vectors, including phage and phagemid vectors

\section{PEOPLE}

Millenium Pharmaceuticals (Cambridge, MA) has named Harry F. Arader Jr. chief financial officer. Arader most recently served as managing director of the greater China division of G.D. Searle \& Co.

Advanced Plant Pharmaceuticals (Monsey, NY) has announced the appointment of Gerard Armand as new clinical trials \& research director. Dr. Armand currently serves as senior associate research scientist for the department of medicine at St. Luke's/Roosevelt Hospital Center located in New York City, as well as president and sci- entific director of GlycoMed Research (Hastings-on-Hudson, NY).

Chiron (Emeryville, CA) has announced that Richard W. Barker has been named senior vice president, and president of Chiron Diagnostics, Chiron's largest business unit. He was previously general manager, worldwide healthcare solutions at IBM Corporation.

Magainin Pharmaceuticals (Plymouth Meeting, PA) has announced the appointment of Thomas J. Bigger as senior vice president, business development, marketing, and sales. Most recently, Mr. Bigger was senior vice president, ethical products of Advanced Polymer Systems.

Celgene Corporation (Warren, NJ) has announced that Robert C. Butler has been appointed senior vice president and chief financial officer. Mr. Butler recently retired as senior vice president and chief financial officer of International Paper Company.

Matrix Pharmaceutical, Inc. (Fremont, CA) has announced the appointment of Sandra 